\title{
Integrated One-Pot Synthesis of 1,3-Oxazinan-2-ones from Isocyanoacetates and Phenyl Vinyl Selenones
}

\author{
Thomas Buyck§, Qian Wang, and Jieping Zhu* \\ §SCS-DSM Award for best poster presentation
}

\begin{abstract}
Brønsted base (Et ${ }_{3} \mathrm{~N}$ or $\left.\mathrm{DBU}\right)$ catalyzed Michael addition of $\alpha$-substituted $\alpha$-isocyanoacetates to phenyl vinyl selenones followed by a Brønsted acid (PTSA) catalyzed domino oxidative cyclization afforded 1,3-oxazinan-2-ones in good to excellent yields. Enantio-enriched 1,3-oxazinan-2-ones were accessible using a Cinchona alkaloid-derived bifunctional catalyst for the first step. In this integrated one-pot process, the phenyl selenonyl group acted consecutively as an activator, a leaving group and a latent oxidant.
\end{abstract}

Keywords: Cinchona - Domino reaction · Isocyanoacetates · Oxidative MCR · Selenones

\section{Introduction}

1,3-Oxazinan-2-one (1) is a scaffold of great interest in many ways. Firstly, this unit is frequently found in compounds displaying antibacterial,[1] antiinflammatory, ${ }^{[2]}$ antidiabetes, ${ }^{[3]}$ and anti-HIV activities. ${ }^{[4]}$ Secondly, this heterocycle is a useful intermediate in the synthesis of pharmaceuticals such as $\operatorname{Prozac}^{\circledR},{ }^{[5]}$ or natural products such as (+)-nagamycin [6] and L-ristosamine, ${ }^{[7]}$ a structural unit of ristomycin belonging to the vancomycin family of antibiotics. ${ }^{[8]}$ Finally, this structural motif is also found in natural substances such as maytansinoids that display potent antitumor activities. ${ }^{[9]}$ From the viewpoint of synthesis, functionalization of homoallylamine (propargylamine) or homoallylic alcohol via halonium-mediated ${ }^{[10]}$ or metal-catalyzed ${ }^{111}$ 6-exo-cyclization, intramolecular 6-exo-Michael addition,[12] intramolecular allylic C-H amination, ${ }^{[13]}$ and tethered aminohydroxylation of olefin $^{[14]}$ are among the most popular methods. We detail herein an operationally sim- ple one-pot synthesis of 4,4-disubstituted 1,3-oxazinan-2-ones 1 from $\alpha$-substituted $\alpha$-isocyanoacetates 2 , phenyl vinyl selenones $\mathbf{3}^{[15,16]}$ and water (Scheme 1). ${ }^{[17]}$ In this integrated one-pot process, the phenyl selenonyl group acted consecutively as an activator, a leaving group and a latent oxidant.

\section{Integrated One-Pot Synthesis of 1,3-Oxazinan-2-ones}

In connection with our continued interest in the chemistry of isocyanoacetate and its derivatives, ${ }^{[18,19]}$ we have recently described an enantioselective synthesis of $\alpha, \alpha$-disubstituted $\alpha$-isocyanoacetates 4. ${ }^{20]}$ When a methanol solution of $\mathbf{4 a}$ was treated with PTSA, a mixture of 1,3-oxazinan-2-one (1a) and 2-methoxy-5,6-dihydro- $4 H$-1,3-oxazine (5a) was produced in a ratio of 4 to 1 . To drive the reaction towards the formation of a single compound 1a, conditions were surveyed by varying the solvents $(\mathrm{MeOH}$, toluene, $t \mathrm{BuOH}$, $t \mathrm{BuOH}-\mathrm{THF}, \mathrm{DMF}$ ), the catalysts (Lewis and Brønsted acids), and temperatures (rt, $\left.35^{\circ} \mathrm{C}, 60^{\circ} \mathrm{C}\right)$. Under optimized conditions (PTSA ( 0.1 equiv) in $t \mathrm{BuOH}$ at $35^{\circ} \mathrm{C}$ ), compound 4a (e.r. 98.1:1.9) was converted into 1a (e.r. 96.8:3.2) in $86 \%$ yield with only a little erosion of the enantiomeric excess.

The scope of this transformation is shown in Scheme 2. The reaction was not affected by the electronic properties of the aromatic ring and heteroarenes such as furan were also tolerated. A single crystal $\mathrm{X}$-ray analysis allowed the confirmation of both the structure and the absolute configuration of compound $\mathbf{1 d}$.

Since PPTS (pyridinium $p$-toluenesulfonate, Scheme 2) is an equally efficient catalyst for the domino oxidative cyclization and the acidity of PTSA ( $\mathrm{pKa}=4.8$ in $\mathrm{H}_{2} \mathrm{O}$ ) is strong enough to protonate most of the Brønsted bases needed to catalyze the Michael addition, we envisioned the direct synthesis of $\mathbf{1}$ from $\mathbf{2}$ and $\mathbf{3}$ by integrating

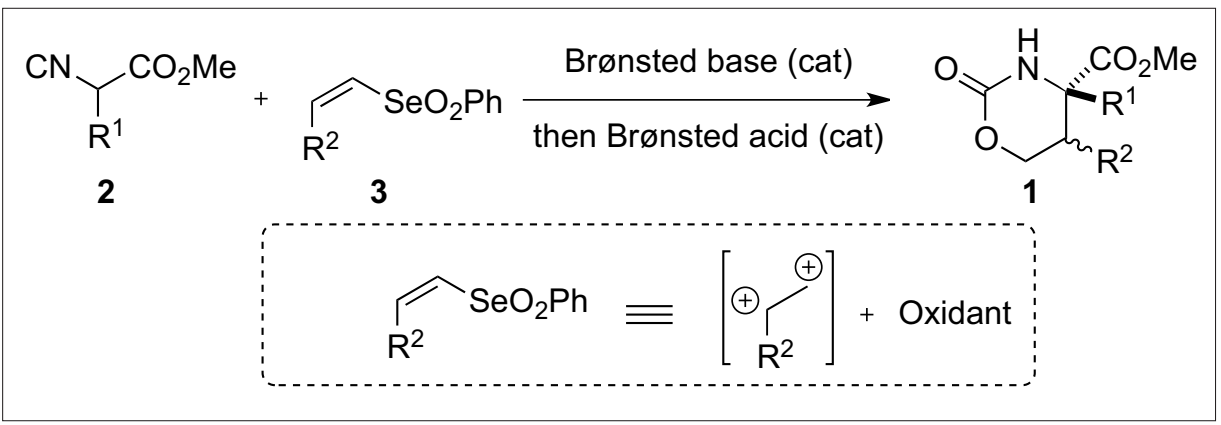




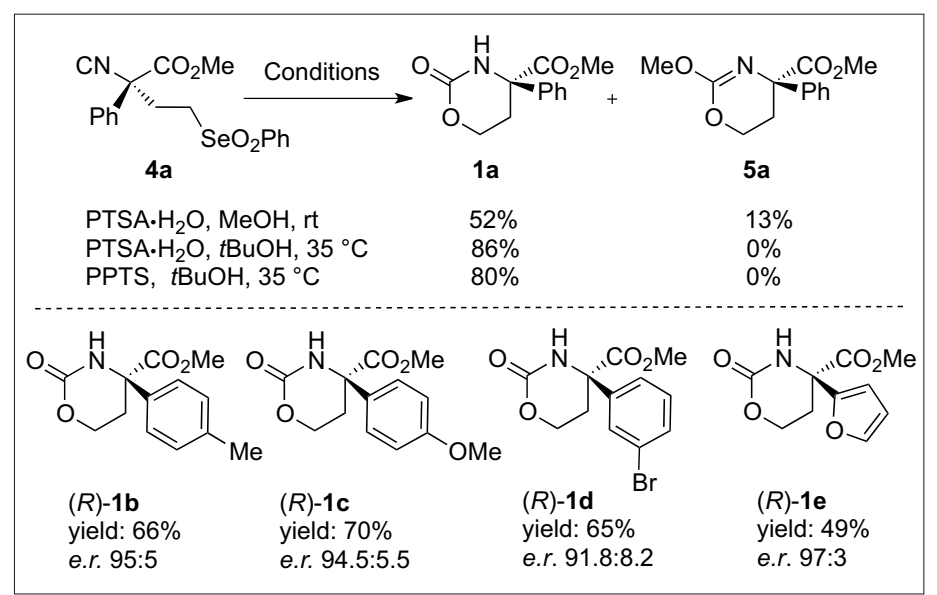

Scheme 2. Oxidative domino cyclization of $\alpha, \alpha$-disubstituted $\alpha$-isocyanoacetates to 1,3-oxazinan2-ones. the two steps into a one-pot process. ${ }^{[21,22]}$ The racemic version was firstly examined using methyl $\alpha$-phenyl- $\alpha$-isocyanoacetate $\mathbf{2 a}\left(\mathrm{R}^{1}=\mathrm{H}\right)$ and $\mathbf{3 a}\left(\mathrm{R}^{2}=\mathrm{H}\right)$ as model substrates (Scheme 3). Indeed, we were able to obtain directly ( \pm )-1a in $75 \%$ yield from $\mathbf{2 a}$ and 3a under optimized conditions $\left(\mathrm{Et}_{3} \mathrm{~N}\right.$ ( 0.1 equiv), $t \mathrm{BuOH}(c \quad 0.25 \mathrm{M})$, rt then PTSA $\cdot \mathrm{H}_{2} \mathrm{O}(0.2$ equiv) in $t \mathrm{BuOH}$, final $c$ $0.05 \mathrm{M}, 35^{\circ} \mathrm{C}$ ). Two equivalents of water were needed to complete the transformation. However, the presence of an excess of water $(5,10,100$ equiv) was not harmful to the reaction.

The scope of this transformation proved to be general (Scheme 3). $\alpha$-Arylated $\alpha$-isocyanoacetates participated efficiently in this reaction delivering the corresponding 4,4-disubstituted 1,3-oxazinan-2-ones $(\mathbf{1 a}-\mathbf{1 j})$ in good yields regardless of the electronic properties of the aryl substituents. The yields are excellent considering that four chemical bonds are formed in this one-pot transformation. The integrated one-pot process was extended to $\alpha$-alkyl substituted $\alpha$-isocyanoacetates by using a stronger base (DBU) for the first step (1k-1n, Scheme 3). The synthetic potential of this methodology was illustrated by converting oxazinanone $\mathbf{1 m}$ into spiropiperidinone (6), the core structure of NK1 antagonists (Scheme 4). [23]

The reaction of $Z$-(prop-1-en-1ylselenonyl)benzene ( $Z-3 \mathbf{b})$ with $2 \mathbf{a}$ delivered 4,4,5-trisubstituted oxazinanone 10 in $75 \%$ yield (d.r. 1:1). A similar reaction between $Z$-3b and $\alpha$-benzyl- $\alpha$ isocyanoacetate afforded $\mathbf{1 p}$ in $58 \%$ yield (d.r. 2:1). Interestingly, no reaction occurred between $E-\mathbf{3 b}$ and $\mathbf{2} \mathbf{a}$ under standard conditions, probably for steric reasons.

Finally, we demonstrated the feasibility of performing this integrated one-pot sequence enantioselectively (Scheme 5). The reaction of $\mathbf{2} \mathbf{e}$ with $\mathbf{3} \mathbf{a}$ in the presence of a catalytic amount of Cinchona alkaloidbased bifunctional organocatalyst 7 followed by addition of a solution of PTSA in ${ }^{t} \mathrm{BuOH}$ furnished $(R)-\mathbf{1 e}$ in $80 \%$ yield with an e.r. of 96.4 to 3.6.

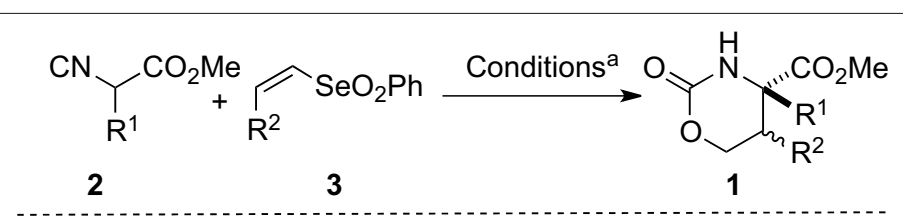<smiles>CC(=O)[C@]1(c2ccc(F)cc2)CCOC(=O)N1</smiles>

1a $F G=H, 75 \%$ 1b $F G=M e, 74 \%$ 1c $F G=O M e, 83 \%$ 1f $F G=F, 72 \%$ FG $1 \mathrm{~g} \mathrm{FG}=\mathrm{Br}, 68 \%$ 1h $1 \mathrm{G}=\mathrm{CF}_{3}, 77 \%$ $1 \mathrm{i} F \mathrm{~F}=\mathrm{NO}_{2}, 65 \%$<smiles>COC(=O)[C@]1(c2cccc(Br)c2)CCOC(=O)N1</smiles>
1d $68 \%$<smiles>COC(=O)[C@]1(c2ccco2)CCOC(=O)N1</smiles><smiles>COC(=O)[C@]1(c2ccoc2)CCOC(=O)N1</smiles>
1j $74 \%$<smiles>COC(=O)[C@]1(Cc2ccccc2)CCOC(=O)N1</smiles>
1e $65 \%$

1 k $74 \%$
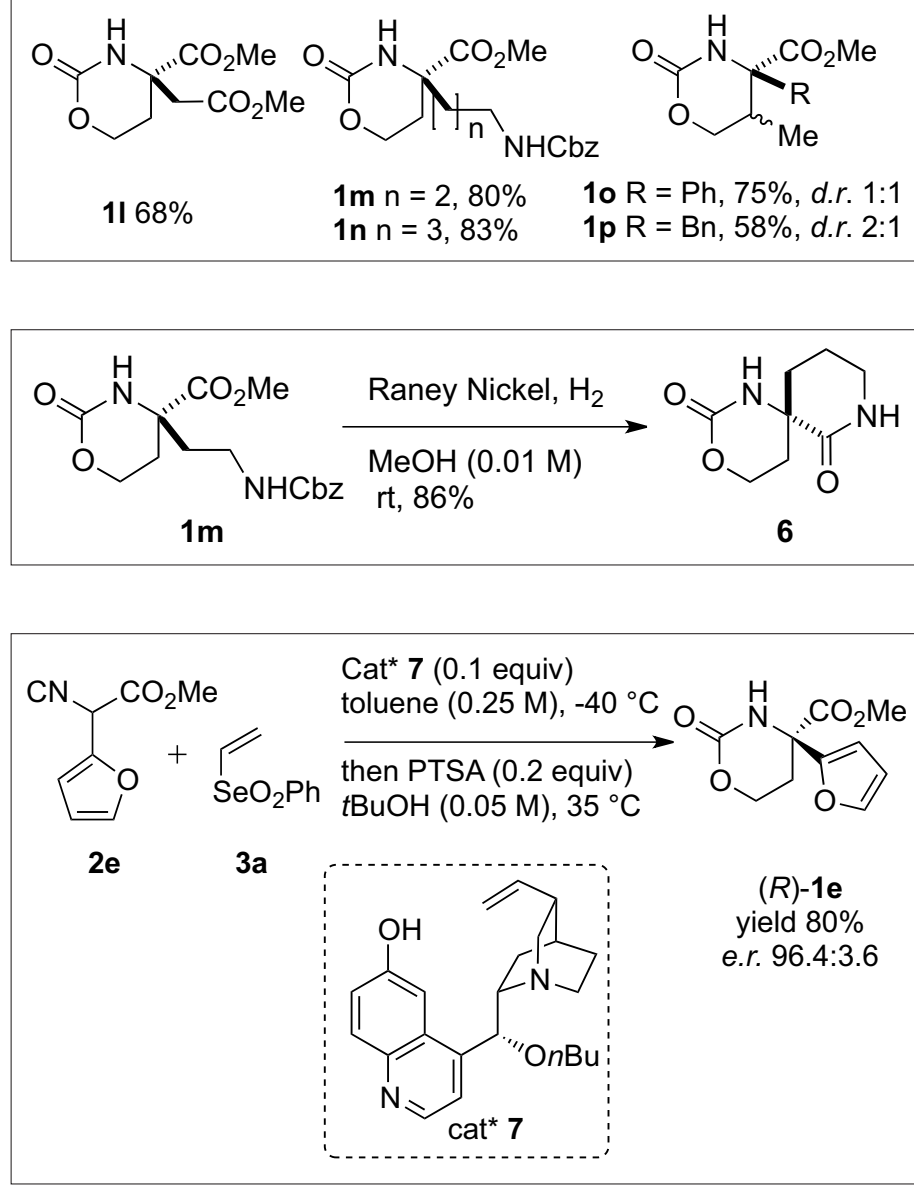

Scheme 4. Access to

spiropiperidinone.

Scheme 3. Scope of the transformation. a: Conditions A for $\mathrm{R}^{1}=$ aryl: $\mathrm{Et}_{3} \mathrm{~N}(0.1$ equiv), $t \mathrm{BuOH}$ (c 0.25 M), rt then PTSA $\bullet \mathrm{H}_{2} \mathrm{O}$ (0.2 equiv), $t \mathrm{BuOH}$ (final c $0.05 \mathrm{M}$ ), 35 ${ }^{\circ} \mathrm{C}$. Conditions $\mathrm{B}$ for $\mathrm{R}^{1}=$ alkyl: DBU (0.05 equiv), $\mathrm{tBuOH}$ (c 0.25 M), rt then PTSA $\bullet \mathrm{H}_{2} \mathrm{O}$ ( 0.1 equiv), $\mathrm{tBuOH}$ (final c $0.05 \mathrm{M}$ ), $35^{\circ} \mathrm{C}$.

Scheme 5. Enantioselective version of the integrated one-pot synthesis of 1,3-oxazinan-2-one. 


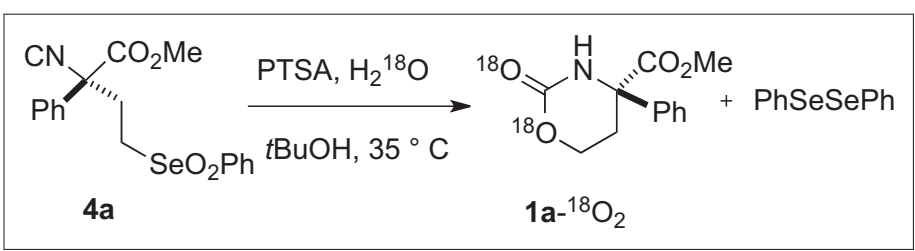

Scheme 6.

${ }^{18} \mathrm{O}$-Labeling experiment. ble ${ }^{18} \mathrm{O}$ incorporation (ratio double:mono $=89: 7$, Scheme 6). Therefore, two molecules of water were participating in the reaction. A shielding effect, in agreement with literature precedents, ${ }^{[24]}$ was observed on the ${ }^{13} \mathrm{C}$ NMR signals of both carbamate carbonyl $(\mathrm{C} 2)$ and $\mathrm{C} 6$ in $\mathbf{1} \mathbf{a}^{18} \mathrm{O}_{2}$ relative to 1a $\left[\Delta \delta_{(\mathrm{C}=\mathrm{O})}{ }^{18}{ }_{\mathrm{O}-}{ }^{16}{ }_{\mathrm{O}}=-3.5 \mathrm{~Hz}, \Delta \delta_{(\mathrm{C} 6)}{ }^{18}{ }_{\mathrm{O}-}{ }^{16}{ }_{\mathrm{O}}=\right.$ $-3.3 \mathrm{~Hz}]$.

The results of these control experiments allowed us to propose a mechanistic pathway as shown in Scheme 7. Hydration of isonitrile under acid catalysis afforded $\mathrm{N}$-alkylformimidic acid $\mathbf{8}$ that is in equilibrium with the corresponding $N$-formamide 9. Intramolecular nucleophilic displacement of phenylselenonyl group by amide oxygen ${ }^{[25]}$ would provide $\mathbf{1 0}$ with the concomitant generation of benzeneseleninic acid that is in equilibrium with benzeneseleninic anhydride (BSA). Acidcatalyzed hydration of $\mathbf{1 0}$ followed by its oxidation by BSA would finally deliver 1,3-oxazinan-2-one $\mathbf{1}$. The oxidation by BSA could proceed either via intermediate $\mathbf{1 2}^{[26]}$ or through the phenylselenation of the nitrogen atom. ${ }^{[27]}$ When methanol was used as solvent, it can compete with water to trap the intermediate $\mathbf{1 0}$ leading to 13 which, upon oxidation by BSA, would deliver 2-methoxy-5,6-dihydro-4H-1,3oxazine 5.[27] The role of benzeneseleninic acid as oxidant was highlighted by the isolation of diphenyldiselenide, its reduced form, from the reaction mixture. Overall, the integrated one-pot process is an oxida- tive multicomponent reaction ${ }^{[28]}$ with an internal redox process. ${ }^{[29]}$

\section{Conclusion}

To sum up, we developed an unprecedented and high yielding one-pot synthesis of 1,3-oxazinan-2-ones from readily available starting materials. The combination of a Brønsted base catalyzed 1,4-addition of $\alpha$-isocyanoacetates to phenyl vinyl selenones followed by a Brønsted acid catalyzed domino oxidative cyclization of the resulting Michael adducts provided the heterocycles with concurrent creation of four chemical bonds. This operationally simple formal four-component $\left(\mathrm{ABC}_{2}\right)$ reaction ${ }^{[28 c, 30]}$ exploits the ability of phenylselenonyl group to act as an activator for Michael addition, a leaving group and a latent oxidant. To the best of our knowledge, such a triple role of phenylselenonyl group is unprecedented in a one-pot transformation.

\section{Acknowledgments}

T. B. would like to cordially thank DSM and Swiss Chemical Society for the SCS-DSM award. We are grateful to EPFL, Swiss National Science Foundation (SNCF), the COST action (CM0905) and Swiss State Secretariat for Education and Research (SER) for financial support.

Received: January 21, 2015
[1] G. Wang, J.-R. Ella-Menye, V. Sharma, Bioorg. Med. Chem. Lett. 2006, 16, 2177.

[2] T. Ullrich, K. Baumann, K. Welzenbach, S. Schmutz, G. Camenisch, J. G. Meingassner, G. Weitz-Schmidt, Bioorg. Med. Chem. Lett. 2004, 14, 2483.

[3] Z. Xu, C. M. Tice, W. Zhao, S. Cacatian, Y.-J. Ye, S.-h B. Singh, P. Lindblom, B. M. McKeever, P. M. Krosky, B. A. Kruk, J. Berbaum, R. K. Harrison, J. A. Johnson, Y. Bukhtiyarov, R. Panemangalore, B. B. Scott, Y. Zhao, J. G. Bruno, J. Togias, J. Guo, R. Guo, P. J. Carroll, G. M. McGeehan, L. Zhuang, W. He, D. A. Claremon, J. Med. Chem. 2011, 54, 6050.

[4] S. D. Young, S. F. Britcher, L. O. Tran, L. S. Payne, W. C. Lumma, T. A. Lyle, J. R. Huff, P. S. Anderson, D. B. Olsen, S. S. Carrol, D. J. Pettibone, J. A. O. Brien, R. G. Ball, S. K. Balani, J. H. Lin, I.-W. Chen, W. A. Schleif, V. V. Sardana, W. J. Long, V. W. Byrnes, E. A. Emini, Antimicrob. Agents Chemother. 1995, 39, 2602.

[5] J. W. Hilborn, Z.-H. Lu, A. R. Jurgens, Q. K. Fang, P. Byers, S. A. Wald, C. H. Senanayake, Tetrahedron Lett. 2001, 42, 8919.

[6] Y.-F. Wang, T. Izawa, S. Kobayashi, M. Ohno, J. Am. Chem. Soc. 1982, 104, 6465.

[7] M. Hirama, T. Shigemoto, S. Itô, J. Org. Chem. 1987, 52, 3342.

[8] a) K. C. Nicolaou, C. N. C. Boddy, S. Bräse, N. Winssinger, Angew. Chem. Int. Ed. 1999, 38, 2096; b) J. Zhu, Expert. Opin. Ther. Pat. 1999, 9, 1005; c) R. D. Süssmuth, ChemBioChem. 2002, 3, 295.

[9] a) S. M. Kupchan, Y. Komoda, W. A. Court, G. J. Thomas, R. M. Smith, A. Karim, C. J. Gilmore, R. C. Haltiwanger, R. F. Bryan, J. Am. Chem. Soc. 1972, 94, 1354; b) F. Taft, K. Harmrolfs, I. Nickeleit, A. Heutling, M. Kiene, N. Malek, F. Sasse, A. Kirschning, Chem. Eur. J. 2012, 18, 880 ; c) For a review, see: J. M. Cassady, K. K. Chan, H. G. Floss, E. Leistner, Chem. Pharm. Bull. 2004, 52, 1 .

[10] M. Fujita, O. Kitagawa, T. Suzuki, T. Taguchi, J. Org. Chem. 1997, 62, 7330.

[11] a) R. Robles-Machín, J. Adrio, J. C. Carretero, J. Org. Chem. 2006, 71, 5023; b) B. Alcaide, P. Almendros, M. T, Quirós, I. Fernández, Beils. J. Org. Chem. 2013, 9, 818.

[12] M. Hirama, T. Shigemoto, Y. Yamazaki, S. Itô, J. Am. Chem. Soc. 1985, 107, 1797.

[13] G. T. Rice, M. C. White, J. Am. Chem. Soc. 2009, 131, 11707.

[14] T. J. Donohoe, C. J. R. Bataille, W. Gattrell, J. Kloesges, E. Rossignol, Org. Lett. 2007, 9, 1725.

[15] a) M. Tiecco, D. Chianelli, L. Testaferri, M. Tingoli, D. Bartoli, Tetrahedron 1986, 42, 4889; b) F. Marini, S. Sternativo, F. Del Verme, L. Testaferri, M. Tiecco, Adv. Synth. Catal. 2009, 351, 103; c) F. Marini, S. Sternativo, F. Del Verme, L. Testaferri, M. Tiecco, Adv. Synth. Catal. 2009, 351, 1801; d) T. Zhang, L. Cheng, S. Hameed, L. Liu, D. Wang, Y.-J. Chen, Chem. Commun. 2011, 47, 6644; e) S. Sternativo, O. Walczak, B. Battistelli, L. Testaferri, F. Marini, Tetrahedron 2012, 68, 10536; f) T. Buyck, Q. Wang, J. Zhu, Angew. Chem. Int. Ed. 2013, 52, 12714 .

[16] For the use of phenyl vinyl selenone as an ethane dication equivalent, see: a) M. Shimizu, I. Kuwajima, J. Org. Chem. 1980, 45, 4063; b) M. Shimizu, R. Ando, I. Kuwajima, J. Org. Chem. 1981, 46, 5246; c) I. Kuwajima, R. Ando, T. Sugawara, Tetrahedron Lett. 1983, 24, 4429; d) R. Ando, T. Sugawara, I. Kuwajima, J. Chem. Soc., Chem. Commun. 1983, 1514; e) M. Shimizu, R. Ando, I. Kuwajima, J. Org. Chem. 1984, 49, 1230; f) R. Ando, T. Sugawara, M. Shimizu, I. Kuwajima, Bull. Chem. Soc. Jpn. 1984, 57, 2897; g) T. Sugawara, I. Kuwajima, 
Tetrahedron Lett. 1985, 26, 5571; h) J. C. Wu, J. Chattopadhyaya, Tetrahedron 1989, 45, 4507; i) W. Jin-Chang, J. Chattopadhyaya, Tetrahedron 1990, 46, 2587; j) W. Tong, J. C. Wu, A. Sandström, J. Chattopadhyaya, Tetrahedron 1990, 46, 3037; k) W. Tong, Z. Xi, C. Gioeli, J. Chattopadhyaya, Tetrahedron 1991, 47, 3431 ; 1) L. Bagnoli, C. Scarponi, L. Testaferri, M. Tiecco, Tetrahedron: Asymm. 2009, 20, 1506; m) S. Sternativo, F. Marini, F. Del Verme, A. Calandriello, L. Testaferri, M. Tiecco, Tetrahedron 2010, 66, 6851; n) L. Bagnoli, C. Scarponi, M. G. Rossi, L. Testaferri, M. Tiecco, Chem. Eur. J. 2011, 17, 993; o) S. Sternativo, A. Calandriello, F. Costantino, L. Testaferri, M. Tiecco, F. Marini, Angew. Chem. Int. Ed. 2011, 50, 9382; p) L. Bagnoli, S. Casini, F. Marini, C. Santi, L. Testaferri, Tetrahedron 2013, 69, 481; q) S. Sternativo, B. Battistelli, L. Bagnoli, C. Santi, L. Testaferri, F. Marini, Tetrahedron Lett. 2013, 54, 6755

[17] T. Buyck, Q. Wang, J. Zhu, J. Am. Chem. Soc. 2014, 136, 11524.

[18] a) D. Bonne, M. Dekhane, J. Zhu, Angew. Chem. Int. Ed. 2007, 46, 2485; b) C. Lalli, M. J. Bouma, D. Bonne, G. Masson, J. Zhu, Chem. Eur. J. 2011, 17, 880.

[19] a) J. Zhu, Eur. J. Org. Chem. 2003, 1133; b) A V. Gulevich, A. G. Zhdanko, R. V. Orru, V. G. Nenajdenko, Chem. Rev. 2010, 110, 5235.

[20] T. Buyck, Q. Wang, J. Zhu, Angew Chem. Int. Ed. 2013, 52, 12714.

[21] For a review, see: J.-I. Yoshida, K. Saito, T. Nokami, A. Nagaki, Synlett, 2011, 1189.
[22] a) C. Piemontesi, Q. Wang, J. Zhu, Org. Biomol. Chem. 2013, 11, 1533; b) Z. Xu, Q. Wang, J. Zhu, Angew. Chem. Int. Ed. 2013, 52, 3272; c) Z. Xu, Q. Wang, J. Zhu, J. Am. Chem. Soc. 2013, 135, 19127; d) O. Wagnières, Z. Xu, Q. Wang, J. Zhu, J. Am. Chem. Soc. 2014, 136, 15102.

[23] S. Paliwal, G. A. Reichard, C. Wang, D. Xiao, H.-C. Tsui, N.Y. Shih, J. D. Arredondo, M. L. Wrobleski, A. Palani, WO 03/051840.

[24] For the few reports dealing with the shielding effect of ${ }^{18} \mathrm{O}$ on the ${ }^{13} \mathrm{C}$ NMR spectroscopy, see: a) J. C. Vederas, J. Am. Chem. Soc. 1980, 102, 374; b) J. M. Risley, R. L. Van Etten, J. Am. Chem. Soc. 1980, 102, 4609; c) Y. Odabachian, S. Tong, Q. Wang, M.-X. Wang, J. Zhu, Angew. Chem. Int. Ed. 2013, 52, 10878.

[25] a) A. Toshimitsu, C. Hirosawa, S. Tanimoto, S. Uemura, Tetrahedron Lett. 1992, 33, 4017; b) A. Toshimitsu, H. Fuji, Chem. Lett. 1992, 21, 2017.

[26] a) D. H. R. Barton, A. G. Brewster, R. A. H. F. Hui, D. J. Lester, S. V. Ley, T. G. Back, J. Chem. Soc., Chem. Commun. 1978, 952; b) M. Shimizu, I. Kuwajima, Tetrahedron Lett. 1979, 20, 2801.

[27] D. H. R. Barton, X. Lusinchi, P. Milliet, Tetrahedron 1985, 41, 4727.

[28] Selected examples of oxidative MCR involving isonitrile, see: a) T. Ngouansavanh, J. Zhu, Angew. Chem. Int. Ed. 2006, 45, 3495; b) T. Ngouansavanh, J. Zhu, Angew. Chem. Int. Ed. 2007, 46, 5775; c) F. Leon, D. G. Rivera, L. A. Wessjohann, J. Org. Chem. 2008, 73, 1762; d) N. Shapiro, A. Vigalok, Angew. Chem. Int. Ed. 2008, 47, 2849; e) G. Jiang, J. Chen, J.-S. Huang, C.-M. Che, Org. Lett. 2009, 11, 4568; f) L. El Kaim, L. Grimaud, J. Oble, S. Wagschal, Tetrahedron Lett. 2009, 50, 1741; g) F. De Moliner, S. Crosignani, L. Banfi, R. Riva, A. Basso, J. Comb. Chem. 2010, 12, 613; h) X. Ye, C. Xie, Y. Pan, L. Han, T. Xie, Org. Lett. 2010, 12, 4240; i) J. Brioche, G. Masson, J. Zhu, Org. Lett. 2010, 12, 1432; j) F. De Moliner, S. Crosignani, A. Galatini, R. Riva, A. Basso, ACS Comb. Sci. 2011, 13, 453; k) X. Ye, C. Xie, R. Huang, J. Liu, Synlett 2012, 409; 1) M. Rueping, C. Vila, Org. Lett. 2013, 15, 2092; m) F. Drouet, G. Masson, J. Zhu, Org. Lett. 2013, 15, 2854; n) B. Karimi, E. Farhangi, Adv. Synth. Catal. 2013, 355, 508.

[29] Oxidative MCR with an internal redox process, see: a) D. Bonne, M. Dehkane, J. Zhu, J. Am. Chem. Soc. 2005, 127, 6926; b) J. M. Grassot, G. Masson, J. Zhu, Angew. Chem. Int. Ed. 2008, 47, 947.

[30] Selected examples of $\mathrm{ABC}_{2}$-type four-component reaction involving isocyanide, see: a) P. Janvier, M. Bois-Choussy, H. Bienaymé, J. Zhu, Angew. Chem. Int. Ed. 2003, 42, 811; b) L. A. Wessjohann, B. Voigt, D. G. Rivera, Angew. Chem. Int. Ed. 2005, 44, 4785; c) G. Rivera, L. A. Wessjohann, J. Am. Chem. Soc. 2006, 128, 7122; d) For a review, see: L. A. Wessjohann, D. G. Rivera, O. E. Vercillo, Chem. Rev. 2009, 109, 796. 